\title{
Anthrovision
}

Vaneasa Online Journal

\section{De la légitimité de l'observateur-filmant}

\author{
Christian Lallier
}

\section{(2) OpenEdition \\ Journals}

Édition électronique

URL : http://journals.openedition.org/anthrovision/1933

DOI : 10.4000/anthrovision.1933

ISSN : 2198-6754

Éditeur

VANEASA - Visual Anthropology Network of European Association of Social Anthropologists

Référence électronique

Christian Lallier, «De la légitimité de l'observateur-filmant », Anthrovision [En ligne], 3.2 | 2015, mis en ligne le 23 août 2016, consulté le 23 avril 2019. URL : http://journals.openedition.org/ anthrovision/1933 ; DOI : 10.4000/anthrovision.1933

Ce document a été généré automatiquement le 23 avril 2019

(c) Anthrovision 


\title{
De la légitimité de l'observateur- filmant
}

\author{
Christian Lallier
}

1 Lorsque j'enseignais - notamment à l'EHESS - la pratique de l'anthropologie filmée, je me souviens des étudiants qui revenaient de leurs exercices de terrain, m'expliquant leurs difficultés rencontrées en raison de la gêne des personnes à être filmées. En écoutant leurs commentaires et en visionnant avec eux leurs images, je m'apercevais rapidement avec eux que ce n'était pas tant les personnes filmées qui éprouvaient un malaise que les élèves eux-mêmes en tant qu'observateur-filmant. On sait discuter longuement des effets de la présence de la caméra sur le comportement des personnes filmées et sur le déroulement des situations observées, mais il conviendrait tout autant de s'intéresser de près à l'embarras que provoque l'acte de filmer lorsque nous nous retrouvons avec une caméra et, parfois, avec un équipement de prise de son, dans une situation qui n'est pas vécue pour être filmée et représentée : au sens où, initialement, la situation ne résulte pas d'un script de mise en scène élaborée et prévue pour les besoins d'un film.

Or, c'est précisément tout l'intérêt de ces trois articles que de mettre en évidence l'embarras du chercheur confronté à l'incongruité de sa présence dans la situation qu'il observe et qu'il filme : en effet, l'observateur-filmant n'a pas de raison d'être là, tant il est vrai que s'il n'était pas là la situation se déroulerait quand même. Il en serait sans doute tout autant si le chercheur n'avait pas de caméra : la situation se déroulerait également en son absence. Sauf que le dispositif filmique met la singularité de la présence de l'observateur en représentation : en d'autres termes, l'observateur est observé par ceux-là mêmes qu'il observe. Mieux, il se retrouve impliqué dans la situation qu'il observe. Là où d'habitude le chercheur reste en retrait sur le terrain avec son bloc notes, et se montre discret derrière son ordinateur, l'observateur-filmant doit suivre au plus près les échanges entre les acteurs qui y sont impliqués afin de rendre compte d'une situation par l'observation filmée.

3 A propos de son terrain sur les actions protestataires à Moscou, Perrine Poupin note que « la caméra oblige le vidéaste-chercheur à être à l'intérieur du site manifestant à partir duquel il observe les interactions entre les participants ». De fait, la situation observée 
devient, selon Perrine Poupin, le «lieu de l'engagement mutuel et premier de l'[ethnographe vidéaste] sur le terrain ». Tout semble ainsi aller de soi, tant que l'observateur-filmant peut rester hors-cadre. Autrement dit, dans la mesure où il ne constitue pas un interlocuteur potentiel pour les personnes observées et filmées. Or, c'est là que tout se complique: en effet, si la caméra permet au chercheur de s'immiscer dans l'intimité sociale des relations qu'il observe, le dispositif filmique (en retour) expose - exhibe - l'observateur dans une mise en représentation de soi à laquelle il doit faire face : en termes goffmaniens, on pourra dire qu'il doit tenir son rôle vis-à-vis des personnes qu'il observe et qu'il filme selon le statut que lui confère l'ordre de l'interaction dans laquelle il est engagé comme observateur-filmant.

Cette mise en représentation expose potentiellement l'observateur-filmant à toutes les sollicitations de la part des personnes filmées. Dans son article sur l'usage des images, de l'argent et de la sorcellerie à Huautla de Jimenez, Marcos Garcia de Teresa décrit ainsi dans le détail comment il se retrouve engagé dans des relations de service auprès des personnes qu'il filme. Il indique tout d'abord que "[ses] hôtes [le] présentaient en plaisantant comme le photographe de la famille ». Mais la plaisanterie dit également, sous le couvert de la blague, le lien de familiarité et de dépendance qui allait se nouer: l'intérêt de ses enregistrements ne manque pas de susciter les convoitises. Prenons par exemple le visionnage des courses de chevaux qu'il a filmées à la demande : pour les uns, il s'agit simplement de revivre la course ; mais pour d'autres, la vérification par l'image permet de lever un doute, voire de servir de manifestation de la preuve, ou encore d'évaluer la performance des chevaux. A l'inverse, parfois, on lui demande de retirer telle image, par crainte qu'elle puisse servir de support à un acte de sorcellerie. Cette situation d'emprise, produite par les relations filmant-filmés, va s'amplifier lorsqu'il réalise les vidéos sur le cheval d'un certain Casimiro, «El Malvado»: ses propres images se retrouvent utilisées dans une campagne électorale d'une mairie locale. L'implication de Marcos Garcia de Teresa en tant qu'observateur-filmant est alors totale, de sorte que la relation entre filmant et filmés constitue le lieu même de son engagement comme ethnographe sur son terrain.

6 De même, Frédérique Leresche, dans son travail sur les musiciens et leur entourage, explique comment elle « devenai[t] une interlocutrice à part entière, la caméra devenant le sujet de [leur] conversation ». Elle reprend un peu plus loin ce constat en ajoutant que ce statut d'interlocutrice à part entière provient du fait qu'elle détient «aussi un outil technique »: le dispositif filmique, la caméra, la rend non seulement visible mais compréhensible. C'est également ce que dit Marcos Garcia de Teresa : « Le fait d'avoir une caméra vidéo a rendu plus compréhensible la raison de [son] séjour sur le terrain pour [ses] enquêtés ». Pour reprendre une terminologie des ethnométhodologues, l'acte de filmer rend prévisible le chercheur sur son terrain : une "prévisibilité » (accountability) qui autorise un ordre social dans la relation d'enquête.

7 Les contributions notent de quelle façon cette caméra permet aux auteurs d'établir une relation sociale avec les personnes filmées. Frédérique Leresche explique tout au long de son article les dilemmes auxquelles elle s'est confrontée en passant du statut de «partenaire de jeu », à celui d'« ethnographe à la caméra ». Perrine Poupin explique de quelle façon sa pratique filmique a légitimé sa présence parmi les manifestants au sens où pour eux «le fait d'être filmé [...] constitue un acte de reconnaissance sur la scène protestataire et médiatique ». On a également vu combien la caméra de Marco Garcia de Teresa l'a introduit dans l'intimité sociale des relations politiques des personnes qu'il 
filme. En fait, tout dispositif technique produit un espace politique et ce qui apparait évident, à travers ces articles, c'est bien que le dispositif filmique produit un espace politique singulier sur le terrain de l'enquête. Mais, cet espace politique peut varier considérablement selon la place occupée par ce dispositif filmique.

Si la prévisibilité permet donc de construire la relation sociale, celle-ci peut aisément se traduire par l'identification de l'observateur-filmant à la figure typique de son activité : comme l'image du pêcheur posant en tenue de pêcheur, le menuisier en tenue de menuisier, le curé en tenue de curé, voilà l'image de l'enquêteur-filmeur avec l'instrument qui le caractérise, s'exposant au regard de ceux-là mêmes qu'il observe. Dès lors, le risque d'être instrumentalisé est grand: autrement dit, d'être intégré au groupe en tant que l'on maîtrise un instrument convoité pouvant servir aux personnes filmées. Marcos Garcia de Terersa devient, dans une certaine mesure, l'obligé des personnes qu'il filme. De même la caméra de Perrine Poupin fut perçue comme un faire-valoir de l'action militante, à tel point que sa «prise d'images n'a pas fait l'objet de débat ni dans les réunions ni dans les discussions informelles ». Elle en donne l'explication un peu plus loin en précisant: «d'une certaine manière, disent les militants, sans ces images, l'action n'existerait pas ». Ici se pose toute la question de la relation entre médias et politique. En l'espèce, Marcos Garcia de Teresa et Perrine Poupin doivent rendre compte des activités politiques tout en faisant preuve de réflexivité quant à l'usage de leurs images qui mettent en représentation ces dites activités.

9 De son côté, Frédérique Leresche note que les musiciens qu'elle suit peuvent avoir l'habitude d'être filmés pendant "l'acte de "musiquer" ", de sorte que le fait de les filmer lui permettait par moment d'avoir une activité qui convenait au cadre d'engagement de la situation de répétition des musiciens et lui donnait ainsi une contenance: «Heureusement, j'ai ma caméra et j'ai passé ma journée à les filmer. Je continue donc cette activité qui me donne une certaine légitimité et qui me donne une "occupation". Cet après-midi déjà, j'ai pu naviguer au milieu d'eux avec ma caméra pendant les séances de répétition sans avoir l'impression de me déplacer en "touriste" mais en ayant quelque chose à faire ". Probablement que sans sa caméra, Frédérique Leresche se serait engagée sur l'objet de sa recherche, comme la plupart des anthropologues sur le terrain, donnant ainsi du sens à sa présence comme anthropologue sur le terrain. La caméra semble ici être utilisée comme un pare-engagement (selon un autre terme d'Erving Goffman) : un peu à la manière de celui qui se met à lire son journal dans la rue pour ne pas croiser le regard d'une personne qu'il connait. Tout se passe comme si la caméra permettait d'éviter toute implication dans la circonstance d'engagement des personnes observées et filmées, alors que ce n'est pas le cas.

10 Car la démarche de tout chercheur (y compris avec une caméra) est bien de rendre compte de l'engagement des personnes dans leur action. Mais voilà, avec l'écriture, il est possible de traduire en temps réel ce que l'on observe en une forme interprétative qui donne à comprendre ce qui se joue. En revanche, avec une caméra, nous sommes confrontés à l'effet de représentation du réel de telle sorte que l'on se retrouve rapidement dans un jeu d'images avec ceux que l'on filme : un jeu qui crée une distance avec la circonstance d'engagement des personnes observées puisque c'est la relation à la caméra qui devient l'objet des attentions entre filmant et filmés. Un jeu qui suppose que le chercheur lui-même entretienne une distance avec sa propre caméra, comme si celle-ci n'était pas légitime à produire un savoir d'anthropologue de telle sorte que la caméra est instrumentalisée par le chercheur dans sa relation à l'autre. 

indique que la caméra s'est vite révélée un support essentiel à la dimension genrée de sa relation d'enquête, autrement dit en tant que chercheuse parmi des musiciens masculins ; « la caméra, dans [certains] contexte[s], a donné du sens à [son] activité ». Plutôt que de donner du sens à l'utilisation de sa caméra par son activité d'anthropologue, sa caméra semble être à ses côtés comme une sorte de double. Ainsi, comme l'indique Marcos Garcia de Teresa ; «[il a] toujours été accompagné d'une caméra vidéo », désignant ainsi un rapport d'altérité à la caméra, comme si celle-ci représentait une individualité propre. un autre. Marcos Garcia de Teresa précise que : «[son] objectif n'était pas de réaliser un film documentaire mais d'utiliser la caméra comme un outil d'appui à [sa] recherche anthropologique ». Perrine Poupin indique quant à elle que «c'est l'expérience filmée de la prise d'images, ainsi que la lecture et le montage de tout type d'images, qui [lui]- ont surtout servi dans [sa] recherche" en ajoutant que "c'est une toute autre histoire que de réaliser un long métrage documentaire construit notamment autour d'un récit». Enfin, Frédérique Leresche note au début de son article que «la caméra est intervenue relativement tôt dans le processus de recherche, d'abord comme complément au carnet de notes ", en remarquant également que «cet outil s'est rapidement révélé être un support essentiel à [sa] relation d'enquête ».

Dans leurs articles respectifs, les chercheurs indiquent qu'ils utilisent la caméra comme un « outil d'appui ", " pour observer ", " comme complément au carnet de notes». Or, que signifient ces termes? En quoi cet usage de la caméra définit leur relation documentaire, pour reprendre le titre de l'atelier organisé lors des Rencontres Annuelles d'Ethnographie en 2014 et qui donne lieu à la publication de ce numéro ? Tout d'abord, il semble que les auteurs ne soient pas vraiment dans une relation documentaire, au sens d'une relation de documentariste, mais plutôt dans une relation filmée, ou plus exactement dans une relation filmante. Le dispositif mis en place par cette relation relève d'une technique d'enregistrement servant l'enquête du chercheur sur son terrain: il s'agit donc d'une relation instrumentalisante, au sens où l'observation filmée devient un acte de captation de situations «saisies sur le vif» et qui feront l'objet ensuite d'un décryptage pour en relever les détails qui ne sont pas perceptibles à l'œil nu. Cet acte de captation place le chercheur dans une position de spectateur, et de mise en regard de la situation. La caméra tend alors à produire une image modélisante: une sorte de représentation figurative qui illustrerait un pattern, un type de situations clairement identifiées et dont la caméra permettrait de capturer un spécimen, à l'instar de l'entomologiste attrapant des papillons.

C'est vraisemblablement cette vision réductrice du réel, que pourrait donner l'image ethnographique, qui semble se poser à Marcos Garcia de Teresa lorsqu'il évoque en introduction de son article « comment l'anthropologie a contribué à créer un produit et une demande touristique » à travers l'image iconique de la chamane Maria Sabina devenue non seulement un emblème mais aussi un modèle médiatique du chamane. Nous percevons comment ce rôle de l'anthropologie, au côtés des médias et du tourisme, dans la fabrication d'une image d'authenticité de la pratique chamanique dans cette région du Mexique, a pu faire douter Marcos Garcia de Teresa de sa propre pratique de l'enquête de terrain avec une caméra : en effet, comment cet outil qui produit de l'image, qui met en représentation, ne pourrait-il pas donner à voir systématiquement une figure typique du 
monde, un pattern modélisant de l'autre, selon une mise en scène dont il faut se déprendre?

Marcos Garcia de Teresa raconte comment il aurait pu aisément faire également des images - voire, un film -, qui reprennent cette représentation fabriquée du chamanisme pour le tourisme en bénéficiant d'une visite guidée de ses hôtes. Mais, plutôt que d'instrumentaliser le réel avec sa caméra, il se laisse instrumentaliser par le réel avec sa caméra. En d'autres termes, il inverse la relation. D'habitude, lorsque l'on fait l'image de quelqu'un on peut dire c'est mon image : au sens que l'image de cette femme, c'est moi qui l'ait faite. De telle sorte que l'on puisse dire, l'image de l'autre c'est l'image de soi. Ce qui revient à s'approprier l'image de l'autre : formulé de cette façon on pourrait y voir une relation d'emprise. Or, en inversant la relation, Marcos Garcia de Teresa devient luimême [le] sujet de ses propres images, au double sens du terme: en tant qu'il en est le motif $^{1}$ et qu'il y est assujetti. L'image qu'il enregistre - qu'il fabrique - résulte de l'image que l'autre lui demande de faire ou de ne pas faire. Dans ce cas, l'image de soi c'est l'image de l'autre (l'image qui appartient à l'autre). Marcos Garcia de Teresa indique qu'il avait " prévu initialement de travailler sur le tourisme chamanique et la commercialisation des champignons hallucinogènes $"$ : autant dire qu'il savait combien il risquait de reproduire l'image du folklore local, cette représentation convenue fabriquée par l'anthropologie et qu'il a justement dénoncée. Par cette inversion, Marcos Garcia de Teresa a trouvé le moyen de se déprendre du syndrome « Maria Sabrina ».

Pour Frédérique Leresche, l'instrumentalisation de la caméra s'est jouée à un autre niveau de la relation d'enquête même si, en définitive, c'est aussi pour se dépendre d'une imagerie typique dans laquelle elle pouvait être enfermée : celle de la femme groupie d'un groupe de musiciens masculins. La caméra lui permettait ainsi de trouver sa place dans le groupe. Elle interprète l'acte de filmer comme un processus de socialisation : l'action de filmer se retrouvait au centre de l'interaction qu'elle était précisément en train de filmer. Là aussi, on retrouve ce processus d'inversion: tout se passe comme si le fait d'instrumentaliser la caméra pour construire sa relation aux autres, le chercheur devenait l'objet même de la situation filmée. Frédérique Leresche souligne la manière dont les musiciens pouvaient jouer avec sa présence, soit « sur le ton de la plaisanterie ou de la complicité » soit en "jouant à cache-cache avec la caméra » ou pour les femmes musiciennes et de l'entourage ; «c'est d'abord dans une sorte de sororité de genre que ce sont faites les négociations à propos de l'usage de la caméra ». Là aussi, son rapport à l'image (à ses images) la lie aux autres par le rapport qu'ils entretiennent eux-mêmes avec leurs images (se mettre en scène).

Selon cette même pratique du terrain ethnographique, Perrine Poupin utilise la caméra comme un outil d'aide à l'enquête, comme un appareil d'enregistrement de données. Mais la caméra lui permet également de se lier avec les manifestants, dans la mesure où ils sont eux-mêmes à la recherche d'une médiatisation de leurs actions. Perrine Poupin ajoute que : « cette expérience filmée [lui] a également permis d'alimenter [sa] recherche depuis une position de vidéaste sur les images produites et diffusées par les militants ». Elle décrit ainsi sa pratique filmique comme une forme d'instrumentalisation du rapport filmant-filmé contribuant à la construction de sa relation d'enquête.

L'examen de ces trois pratiques de terrain rappelle que tout dispositif technique doit faire l'objet d'une traduction symbolique afin d'être utilisé : en d'autres termes, un instrument pourra s'insérer dans l'économie d'une situation sociale, dès lors qu'il correspondra à une valeur des échanges. L'utilisation d'un instrument de musique, par exemple, (son 
instrumentalisation, sa manipulation) se traduira par la production mélodique et/ou rythmique qu'il procure. Mais, il peut également faire l'objet d'une instrumentalisation (d'une manipulation) des rapports entre les musiciens; son acquisition peut se traduire par un jeu d'influence entre les partenaires d'une transaction marchande. De même, la pratique filmique peut servir à la construction d'une relation d'enquête par l'instrumentalisation du rapport filmant-filmé.

Mais, la notion de manipulation induit une volonté d'influer et de dominer, ce qui n'est évidemment pas le cas des pratiques d'enquêtes présentées lors de ces Rencontres Ethnographiques. Non seulement les chercheurs n'ont pas l'intention d'influer sur les personnes filmées, mais ils ont conscience que leur pratique filmique peut créer un biais à leur enquête de telle sorte qu'ils s'attachent à se déprendre de cette emprise technique en utilisant la caméra comme une condition d'accès à leur terrain. Tout se passe comme si, cette utilisation de la caméra comme valorisation de la relation d'enquête permettait au chercheur de légitimer à la fois sa présence sur le terrain et sa possibilité de filmer. Mais, ce faisant, les chercheurs ne se reconnaissant pas une pleine légitimité à rendre compte des situations observées par l'observation filmée des interactions sociales: pour cela, il conviendrait de s'attacher tout particulièrement à l'engagement des personnes dans leur action, ce qui suppose précisément de ne pas y inclure le rapport filmant-filmé lui-même.

\section{NOTES}

1. Ces images qu'il fait des autres sont bien des images de lui.

\section{RÉSUMÉS}

Il nous semble si aisé de filmer que bien souvent nous estimons que ce sont les personnes filmées qui sont gênées par la présence de la caméra. Mais, en fait, la plus grande difficulté c'est bien d'accepter l'incongruité de sa propre place d'observateur-filmant: autrement dit, de savoir « être là » alors même que les acteurs de la situation n'ont pas besoin de notre présence. Dès lors, la production des images peut devenir l'objet d'une économie de l'échange entre filmant et filmés, au risque de menacer le «terrain » et l'enquête du chercheur.

We are now so at ease when we film that we often consider the subjects of the film to be the most embarrassed by the presence of the camera. But in fact the greatest difficulty is to recognise the incongruity of the place of the filming observer: in other words, to know how to 'be there' although the actors in the situation do not need our presence. In these terms, the production of images can become the object of an economy of exchange between the filmer and the filmed, at the risk of threatening the 'territory' and research of the researcher. 
El hecho de filmar nos parece tan simple que a menudo consideramos que son las personas filmadas las que se encuentran incómodas por la presencia de la camara. Sin mebargo, la gran dificultad reside en aceptar la incongruencia de el posicionamiento del observador-filmante: dicho en otras palabras, se saber "estar allí" a pesar de que los actores de la situación no precisan de nuestra presencia. A partir de este momento, la producción de imágenes puede convertirse en el objeto de una economía del intercambio entre filmante y filmados, con el riesgo, eso sí, de alterar el "terreno" y la investigación del investigador o investigadora.

INDEX

Mots-clés : relation filmante, dispositif filmique, caméra, pouvoir, instrumentalisation

Keywords : filming relation, filmic apparatus, camera, power, instrumentalization

Palabras claves : Relación filmante, dispositivo fílmico, camara, poder, intrumentalización

\section{AUTEUR}

\section{CHRISTIAN LALLIER}

Anthropologue-Cinéaste, Chercheur associé au IIAC-LAU (UMR 8177). Institut Interdisciplinaire d'Anthropologie du Contemporain - Laboratoire d'Anthropologie Urbaine 